\title{
Infarto Agudo do Miocárdio Complicado por Choque Cardiogênico: Efeito da Circulação Colateral nos Resultados da Intervenção Coronária Percutânea Primária - Dados do Registro InCor
}

\author{
Leandro Richa Valim¹, Augusto Celso A. Lopes ${ }^{1}$, Igor Ribeiro de Castro Bienert ${ }^{1}$, Henrique B. Ribeiro' \\ Carlos A. Campos ${ }^{1}$, Rodrigo B. Esper ${ }^{1}$, Silvio Zalc ${ }^{1}$, Marco A. Perin¹, Pedro A. Lemos ${ }^{1}$, \\ Expedito E. Ribeiro ${ }^{1}$, José Antonio F. Ramires ${ }^{1}$
}

\section{RESUMO}

Introdução: A presença de circulação colateral no contexto do infarto agudo do miocárdio (IAM) pode exercer um fator de proteção, levando a menor área de infarto e melhor função ventricular. Este trabalho procurou examinar as características clínicas dos pacientes com diferentes graus de circulação colateral e a influência desta na evolução do IAM com supradesnivelamento de segmento ST (IAMCSST) complicado com choque cardiogênico à admissão hospitalar e tratados com intervenção coronária percutânea (ICP) primária. Métodos: Registro unicêntrico que realizou seguimento prospectivo no período de 2001 a 2009, incluindo 105 pacientes divididos em dois grupos, de acordo com o grau de circulação colateral: grupo 1, circulação colateral graus $0 / 1 \quad(n=83)$; e grupo 2 , circulação colateral graus $2 / 3(n=22)$. As características clínicas e angiográficas e os desfechos hospitalares foram comparados entre os grupos. Resultados: Ambos os grupos foram semelhantes em relação às características clínicas, exceto pela menor média de idade $(65,9$ anos vs. 57,8 anos; $P=0,015)$ e maior tendência ao tabagismo atual no grupo com colateral graus $2 / 3(15,7 \%$ vs. $31,8 \% ; P=0,08)$. Quanto às características angiográficas, nos indivíduos com colateral graus $2 / 3$ houve maior frequência de lesões/paciente (1,3 lesão vs. 1,8 lesão; $\mathrm{P}=0,02)$ e maior acometimento de bifurcação coronária $(18,5 \%$ vs. $36,4 \%$; $P=0,03)$. Na evolução intra-hospitalar ficou evidenciada menor mortalidade nos pacientes com circulação colateral exuberante $(49,4 \%$ vs. $27,3 \%$, risco relativo de 0,$55 ; \mathrm{P}=0,05)$. Conclusões: Em pacientes com IAMCSST e choque cardiogênico tratados com ICP primária a presença de circulação

\section{ABSTRACT}

Acute Myocardial Infarction Complicated by Cardiogenic Shock: Effect of Collateral Circulation in Primary Coronary Intervention Results Data from the InCor Registry

Background: The presence of collateral circulation in the context of acute myocardial infarction (AMI) may play a protective role, leading to smaller infarction areas and improved ventricular function. This study was aimed at assessing the clinical characteristics of patients with different degrees of collateral circulation and the effect on outcomes of ST segment elevation AMI (STEMI) complicated by cardiogenic shock at hospital admission and treated by primary percutaneous coronary intervention $(\mathrm{PCl})$. Methods: Single center registry with a prospective follow-up from 2001 to 2009, including 105 patients divided into two groups, according to the degree of collateral circulation: Group 1, collateral circulation grades $0 / 1(n=83)$; and Group 2, collateral circulation grades $2 / 3(n=22)$. Clinical and angiographic characteristics and hospital outcomes were compared between groups. Results: The clinical characteristics of both groups were similar, except for a lower mean age (65.9 years vs. 57.8 years; $\mathrm{P}=0.015)$ and a trend to current smoking in the grade $2 / 3$ group $(15.7 \%$ vs. $31.8 \% ; P=0.08)$. As to angiographic characteristics, individuals with grades $2 / 3$ had a higher rate of lesions per patient (1.3 vs. $1.8 ; \mathrm{P}=0.02)$ and more bifurcation lesions (18.5\% vs. $36.4 \%$; $\mathrm{P}=0.03)$. In-hospital outcomes showed lower mortality in patients with good collateral circulation $(49.4 \%$ vs. $27.3 \%$, relative risk $0.55 ; \mathrm{P}=0.05)$.

1 Instituto do Coração do Hospital das Clínicas da Faculdade de Medicina da Universidade de São Paulo (InCor/HCFMUSP) - São Paulo, SP, Brasil.

Correspondência: Leandro Richa Valim. Rua Joaquim Távora, 974/ 113 - Vila Mariana - São Paulo, SP, Brasil - CEP 04015-012 E-mail: leandro.valim@yahoo.com.br

Recebido em: 28/3/2011 • Aceito em: 3/6/2011 
colateral pode favorecer um melhor prognóstico na evolução intra-hospitalar.

DESCRITORES: Angioplastia. Infarto do miocárdio. Choque cardiogênico. Circulação colateral.

E ntre os pacientes com infarto agudo do miocárdio (IAM), o tamanho da área infartada é o principal determinante do remodelamento ventricular e da subsequente mortalidade. ${ }^{1} \mathrm{O}$ tamanho da área infartada, por sua vez, está relacionado à área de miocárdio em risco, que pode sofrer influência da presença ou não de circulação colateral para a região irrigada pela artéria relacionada ao IAM. $^{2}$

Algum grau de circulação colateral é encontrado em cerca de $40 \%$ dos pacientes com IAM. ${ }^{3}$ A circulação colateral pode preceder ou se desenvolver logo após a oclusão arterial ${ }^{4}$, sendo mais comum em pacientes jovens e naqueles com angina prévia. ${ }^{5}$ Entretanto, o impacto da circulação colateral nos desfechos clínicos em pacientes com IAM permanece controverso. Para pacientes com reperfusão tardia, a presença de colaterais tem sido associada a maior viabilidade miocárdica e melhor evolução intra-hospitalar após IAM. ${ }^{5-7}$ Por sua vez, em pacientes submetidos a reperfusão precoce, os estudos que avaliaram a influência da circulação colateral possuem resultados conflitantes. ${ }^{8-11}$

O choque cardiogênico ocorre como complicação em $7 \%$ a $10 \%$ dos casos de IAM. É a principal causa de morte nesses pacientes, estando associado a taxa de mortalidade de $70 \%$ a $80 \% .^{12,13}$ Essa população foi avaliada pelo estudo Should We Emergently Revascularize Occluded Coronaries for Cardiogenic Shock (SHOCK) ${ }^{14}$, no qual 302 pacientes com IAM e choque cardiogênico foram randomizados para revascularização (percutânea ou cirúrgica) de emergência ou estabilização clínica inicial. Apesar de não haver diferença na mortalidade intra-hospitalar, observou-se significativa redução da mortalidade em 6 e 12 meses no grupo de revascularização de emergência. ${ }^{14,15}$

Desde 1999 a estratégia de revascularização precoce nesse contexto é caracterizada como classe de recomendação I pelos consensos da American Heart Association/ American College of Cardiology (AHA/ACC). ${ }^{16}$ A partir daí tem-se observado queda progressiva da mortalidade. Essa tendência tem sido explicitada em registros, como o publicado por Babaev et al. ${ }^{17}$, realizado em 775 centos americanos, com 293.633 pacientes, entre janeiro de 1995 e maio de 2004, que mostrou queda da mortalidade intra-hospitalar de 60,3\% em 1995 para 47,9\% em 2004 ( $P<0,001)$. No entanto, apesar da melhora evolutiva, a mortalidade nesse contexto ainda permanece alta.
Conclusions: In patients with STEMI and cardiogenic shock treated by primary $\mathrm{PCl}$, the presence of collateral circulation may improve in-hospital prognosis.

KEY-WORDS: Angioplasty. Myocardial infarction. Shock, cardiogenic. Collateral circulation.

Trabalhos anteriores sugerem que a presença de circulação colateral pode reduzir a evolução para choque cardiogênico e a mortalidade. ${ }^{3,18}$ São poucos os dados na literatura sobre a influência da circulação colateral especificamente no subgrupo de pacientes com IAM que evoluíram com choque cardiogênico e foram submetidos a intervenção coronária percutânea (ICP) primária. O objetivo deste estudo foi avaliar a influência da presença de circulação colateral, para a área infartada, na evolução intra-hospitalar do grupo de pacientes com IAM com supradesnivelamento do segmento ST (IAMCSST) complicado com choque cardiogênico à admissão hospitalar.

\section{MÉTODOS}

\section{População do estudo}

Foram avaliados no presente estudo os pacientes com diagnóstico de IAMCSST e submetidos a ICP primária em um único centro (Instituto do Coração do Hospital das Clínicas da Faculdade de Medicina da Universidade de São Paulo - InCor/HCFMUSP, São Paulo, SP, Brasil), no período de janeiro de 2001 a maio de 2009. Foram incluídos para análise todos os pacientes que se apresentaram com choque cardiogênico à admissão. Os pacientes foram divididos em dois grupos, de acordo com o grau de circulação colateral pela classificação de Rentrop et al. ${ }^{4}$ : grupo 1 , circulação colateral graus $0 / 1$; e grupo 2 , circulação colateral graus $2 / 3$.

\section{Procedimento}

A utilização de pré-dilatação com balão ou de stent direto ficou a critério do operador. A antiagregação plaquetária utilizada foi clopidogrel em dose de ataque de $300 \mathrm{mg}$ a $600 \mathrm{mg}$, seguido de $75 \mathrm{mg} / \mathrm{dia}$ por pelo menos 30 dias, com recomendação de um ano em casos sem contraindicação. Além disso, o paciente recebeu prescrição para utilizar aspirina (100-300 mg/dia) indefinidamente. Após obtenção de acesso vascular $(6 \mathrm{~F}$ ou 7 F) e introdução do cateter-guia, foi administrada heparina nas doses preconizadas para se obter tempo de coagulação ativada > 250 segundos (ou > 200 segundos se houvesse utilização de inibidores da glicoproteína IIb/IIla).

\section{Coleta e análise de dados}

Os dados da evolução intra-hospitalar foram coletados por médicos treinados, durante a internação 
índice, seguindo o preenchimento de formulários previamente padronizados. A coleta incluiu características clínicas, resultados de exames laboratoriais, dados do procedimento e evolução clínica até a alta hospitalar. Todas as angiografias foram analisadas pelo Serviço de Hemodinâmica e Cardiologia Intervencionista do InCor. A aquisição de imagens foi realizada utilizando-se duas ou mais projeções angiográficas da estenose, após a administração de nitrato.

\section{Definições}

O diagnóstico de IAMCSST foi realizado na ocorrência de elevação persistente de ST > $1 \mathrm{~mm}$ em duas derivações contíguas ou novo bloqueio de ramo esquerdo ao eletrocardiograma.

Para o tipo de lesão, foram utilizados os critérios do American College of Cardiology/American Heart Association. ${ }^{19}$ Para a classificação do fluxo coronário pré e pós-procedimento foi utilizada a classificação TIMI. ${ }^{20}$ Sucesso angiográfico foi definido como lesão residual $<30 \%$, com manutenção ou restabelecimento do fluxo anterógrado normal (TIMI 3). ${ }^{21}$

Choque cardiogênico foi definido por critérios clínicos, conforme o SHOCK Trial, pela presença de hipotensão [pressão arterial sistólica (PAS) $<90 \mathrm{mmHg}$ por pelo menos 30 minutos ou necessidade de medidas de suporte para manter PAS $>90 \mathrm{mmHg}$ ] e hipoperfusão orgânica (débito urinário $<30 \mathrm{ml} /$ hora e frequência cardíaca > 60 bpm). ${ }^{14}$

Para se definir o grau de circulação colateral antes da realização da ICP, seguiu-se a classificação de
Rentrop et al. ${ }^{4}$, previamente citada: grau $0=$ nenhum enchimento colateral visível; $1=$ enchimento de ramos laterais da artéria relacionada ao infarto, sem atingir o segmento epicárdico; 2 = enchimento parcial do vaso epicárdico; e 3 = enchimento completo do vaso colateral promovido pelas colaterais.

\section{Análise estatística}

Os dados foram analisados no programa de estatísticas SPSS versão 17.0. As variáveis contínuas foram descritas como média \pm desvio padrão e foram avaliadas pelo teste $t$ de Student. As variáveis categóricas foram representadas como sua porcentagem, sendo comparadas com o teste de qui-quadrado. O nível de significância adotado foi $P \leq 0,05$.

\section{RESULTADOS}

Durante o período do estudo (janeiro de 2001 a maio de 2009), foram realizadas, no total, 1.053 ICPs primárias. Destas, 105 (10\%) foram em pacientes que apresentavam choque cardiogênico à admissão, sendo incluídos neste estudo. Entre os pacientes analisados, 83 apresentavam circulação colateral graus $0 / 1$ (grupo 1) e 22, circulação colateral graus 2/3 (grupo 2).

Conforme demonstrado na Tabela 1 , os pacientes do grupo 2 eram mais jovens $(57,8 \pm 15,5$ vs. 65,9 \pm $13,2 ; P=0,015)$, não se observando diferenças entre os grupos quanto a sexo, fatores de risco para aterosclerose ou eventos cardíacos prévios. Observou-se tendência a maior tabagismo no grupo 2 (31,8\% vs. 15,7\%; $P=0,08)$.

TABELA 1

Características clínicas basais dos pacientes com infarto agudo do miocárdio e choque cardiogênico submetidos a intervenção coronária percutânea primária

\begin{tabular}{lccc}
\hline & $\begin{array}{c}\text { Grupo } \mathbf{1} \\
(\mathbf{n = 8 3 )}\end{array}$ & $\begin{array}{c}\text { Grupo 2 } \\
(\mathbf{n = 2 2})\end{array}$ & $\mathbf{P}$ \\
\hline Idade, anos & $65,9 \pm 13,2$ & $57,8 \pm 15,5$ & 0,015 \\
Sexo masculino, \% & 66,3 & 63,6 & 0,83 \\
HAS, \% & 73,5 & 68,2 & 0,4 \\
Diabetes, \% & 30,1 & 36,4 & 0,37 \\
Dislipidemia, \% & 39,8 & 27,3 & 0,2 \\
Tabagismo, \% & 15,7 & 31,8 & 0,08 \\
AVC prévio, \% & 3,6 & 0 & 0,57 \\
DPOC, \% & 7,2 & 4,5 & 0,54 \\
IRC dialítica, \% & 4,8 & 9,1 & 0,37 \\
IVP, \% & 8,4 & 4,5 & 0,46 \\
IAM prévio, \% & 25,3 & 27,3 & 0,52 \\
ICC, \% & 20,5 & 31,8 & 0,19 \\
\hline
\end{tabular}

Grupo 1 = circulação colateral graus $0 / 1$; Grupo 2 = circulação colateral graus 2/3.

AVC = acidente vascular cerebral; DPOC = doença pulmonar obstrutiva crônica; HAS = hipertensão arterial sistêmica; IAM = infarto agudo do miocárdio; ICC = insuficiência cardíaca congestiva; IRC = insuficiência renal crônica; IVP = insuficiência vascular periférica; $\mathrm{n}=$ número de pacientes. 
As características angiográficas estão expostas na Tabela 2. Não houve diferenças entre os grupos quanto à extensão da doença coronária ou ao tempo decorrido entre o início da dor e a realização de ICP primária. Observa-se maior número de lesões por paciente no grupo $2(1,8 \pm 0,85$ vs. $1,3 \pm 0,5 ; P=0,02)$, apesar de não haver diferenças quanto ao número de stents utilizados $(1,8 \pm 0,4$ vs. $1,9 \pm 0,6 ; P=0,46)$ ou ao comprimento total dos stents $(26,6 \pm 14,1$ vs. $25,1 \pm 11,2$;
$P=0,61)$. Os pacientes do grupo 2 apresentaram tendência a lesões mais complexas, notando-se maior incidência de lesões envolvendo bifurcações (36,4\% vs. $18,5 \% ; P=0,03)$, de lesões ulceradas $(63,3 \%$ vs. $36,6 \% ; \mathrm{P}=0,09)$, com imagem sugestiva de trombo (78,8\% vs. 66,1\%; $P=0,11)$ e morfologia B2/C $(92,3 \%$ vs. $80,6 \% ; P=0,12)$. Observou-se baixo uso de balão de contrapulsação aórtico, não apresentando diferenças entre os grupos $(17,1 \%$ no grupo 1 vs. $15,8 \%$ no

TABELA 2

Características angiográficas e do procedimento dos pacientes com infarto agudo do miocárdio e choque cardiogênico submetidos a intervenção coronária percutânea primária

\begin{tabular}{|c|c|c|c|}
\hline & $\begin{array}{l}\text { Grupo } 1 \\
(n=83)\end{array}$ & $\begin{array}{l}\text { Grupo } 2 \\
(n=22)\end{array}$ & $\mathbf{P}$ \\
\hline Padrão arterial, \% & & & 0,08 \\
\hline Uniarterial & 27,7 & 18,2 & \\
\hline Biarterial & 21,7 & 45,5 & \\
\hline Triarterial & 50,6 & 36,4 & \\
\hline Total de lesões & $1,3 \pm 0,5$ & $1,8 \pm 0,85$ & 0,02 \\
\hline Tempo desde o início da dor, horas & $6,7 \pm 3,4$ & $5,5 \pm 3,9$ & 0,47 \\
\hline Número de stents & $1,9 \pm 0,6$ & $1,8 \pm 0,4$ & 0,46 \\
\hline Diâmetro do stent, mm & $3,1 \pm 0,44$ & $3,1 \pm 0,5$ & 0,86 \\
\hline Comprimento total dos stents, $\mathrm{mm}$ & $25,1 \pm 11,2$ & $26,6 \pm 14,1$ & 0,61 \\
\hline Uso de BIA, \% & 17,1 & 15,8 & 0,59 \\
\hline Lesão ostial, \% & 21,4 & 30,3 & 0,2 \\
\hline Lesão ulcerada, \% & 36,6 & 63,3 & 0,09 \\
\hline Lesão excêntrica, \% & 67,6 & 65,6 & 0,49 \\
\hline Trombo, \% & 66,1 & 78,8 & 0,11 \\
\hline Bifurcação, \% & 18,5 & 36,4 & 0,03 \\
\hline Morfologia B2/C & 80,6 & 92,3 & 0,12 \\
\hline Fluxo final TIMI III, \% & 96,3 & 100 & 0,67 \\
\hline
\end{tabular}

Grupo 1 = circulação colateral graus $0 / 1$; Grupo 2 = circulação colateral graus $2 / 3$.

$\mathrm{BIA}=$ balão intra-aórtico; $\mathrm{n}=$ número de pacientes .

TABELA 3

Desfechos clínicos intra-hospitalares dos pacientes com IAM e choque cardiogênico submetidos a intervenção coronária percutânea primária

\begin{tabular}{lccc}
\hline & $\begin{array}{c}\text { Grupo 1 } \\
(\mathbf{n = 8 3 )}\end{array}$ & $\begin{array}{c}\text { Grupo 2 } \\
(\mathbf{n = 2 2})\end{array}$ & $\mathbf{P}$ \\
\hline Óbito, \% & 49,4 & 27,3 & 0,05 \\
AVC, \% & 2,4 & 0 & 0,6 \\
Nova ICP, \% & 3,6 & 0 & 0,49 \\
Sangramento maior, \% & 2,4 & 0 & 0,6 \\
Transfusão, \% & 1,2 & 0 & 0,79 \\
IRA, \% & 10,8 & 4,5 & 0,33 \\
\hline
\end{tabular}

Grupo 1 = circulação colateral graus 0/1; Grupo 2 = circulação colateral graus 2/3.

$\mathrm{AVC}=$ acidente vascular cerebral; ICP = intervenção coronária percutânea; IRA = insuficiência renal aguda; $\mathrm{n}=$ número de pacientes. 
grupo $2 ; P=0,59)$. A taxa de sucesso da intervenção coronária percutânea foi alta tanto no grupo 1 como no grupo $2(96,3 \%$ vs. $100 \% ; P=0,67)$

Desfechos clínicos e angiográficos intra-hospitalares estão expostos na Tabela 3. Os grupos foram semelhantes em relação a quase todos os desfechos clínicos de evolução intra-hospitalar, à exceção de mortalidade global, que foi menor no grupo com circulação colateral mais exuberante $[49,4 \%$ vs. $27,3 \%$; razão de risco (RR) 0,55, intervalo de confiança de 95\% (IC 95\%) 0,46-0,90; P = 0,05). Não houve diferenças significativas entre os grupos quanto à ocorrência de acidente vascular cerebral $(2,4 \%$ vs. $0 ; P=0,6)$, necessidade de nova ICP $(3,6 \%$ vs. $0 ; P=0,49)$, ocorrência de sangramento maior $(2,4 \%$ vs. $0 ; P=0,6)$ ou necessidade de hemotransfusão $(1,2 \%$ vs. $0 ; P=0,79)$.

\section{DISCUSSÃO}

O presente estudo teve como objetivo avaliar a influência da presença de circulação colateral na evolução intra-hospitalar de pacientes com IAMCSST, complicado com choque cardiogênico, submetidos a ICP primária. Nesta série os pacientes com circulação colateral mais exuberante eram mais jovens e com maior prevalência de tabagistas, o que vai ao encontro do observado na literatura ${ }^{5}$, não se encontrando, no entanto, explicações fisiopatológicas definitivas para tais correlações. Além disso, neste estudo observou-se maior número de lesões por paciente, o que também foi demonstrado em outros trabalhos. ${ }^{22,23}$

A presença de colateral foi associada a menor mortalidade global intra-hospitalar, apesar de não haver diferenças entre os grupos nas demais características avaliadas. Pérez-Castellano et al. ${ }^{3}$ e Desch et al. ${ }^{18}$ demonstraram que a presença de circulação colateral se apresenta como fator protetor na população global de pacientes com IAM, reduzindo a evolução para choque cardiogênico, entre outros eventos. Apesar da pequena amostra estudada, os resultados deste estudo sugerem que, mesmo naquele subgrupo de pacientes de maior gravidade que se apresentam com choque cardiogênico, a presença de circulação colateral pode também se mostrar como fator protetor, com redução de mortalidade intra-hospitalar.

Um subestudo do Occluded Artery Trial (OAT), publicado recentemente ${ }^{24,25}$, observou que a presença de circulação colateral estava relacionada a menor mortalidade e desenvolvimento de insuficiência cardíaca, mas sem efeito benéfico quanto à incidência de reinfarto. Entretanto, após análise multivariada, tal efeito não se confirmou. Comparando-se esse subestudo com o presente trabalho, observa-se que naquele os pacientes incluídos possuíam perfil de gravidade menor, uma vez que excluiu os pacientes com choque cardiogênico e apresentava elevada proporção de pacientes em Killip I, enquanto esta amostra foi composta exclusivamente por pacientes em choque cardiogênico. Outro fator a ser discutido é o tempo decorrido para a ICP, já que a intervenção no OAT foi realizada em 3 a 28 dias após o IAM, enquanto neste trabalho foram incluídos pacientes submetidos a ICP primária.

Apesar de o tratamento invasivo precoce nos pacientes com IAMCSST e choque cardiogênico ter implementado benefícios, com redução relativa da mortalidade, esta continua elevada, girando em torno de $50 \%$ a $60 \%$. A ausência de circulação colateral para a área infartada poderia ser considerada fator de mau prognóstico a curto prazo em pacientes com IAMCSST e choque cardiogênico.

\section{Limitações do estudo}

As limitações que podem implicar potencial viés na interpretação dos resultados deste estudo foram: 1) natureza observacional e retrospectiva; 2) realização em um único centro; 3) amostra pequena; 4) não-realização de análise da função ventricular esquerda, em decorrência da indisponibilidade desses dados em parcela expressiva dos pacientes; e 5) ausência de seguimento tardio. No entanto, trata-se de uma população selecionada de alta complexidade e pouco explorada na literatura.

\section{CONCLUSÕES}

Os resultados deste estudo sugerem que a presença de circulação colateral no quadro de IAMCSST complicado por choque cardiogênico pode exercer um fator de proteção, levando a melhor prognóstico durante a evolução intra-hospitalar.

\section{CONFLITO DE INTERESSES}

Os autores declaram não haver conflito de interesses relacionado a este manuscrito.

\section{REFERÊNCIAS}

1. Chareonthaitawee P, Christian TF, Hirose K, Gibbons RJ, Rumberger JA. Relation of initial infarct size to extent of left ventricular remodeling in the year after acute myocardial infarction. J Am Coll Cardiol. 1995;25(3):567-73.

2. Christian TF, Schwartz RS, Gibbons RJ. Determinants of infarct size in reperfusion therapy for acute myocardial infarction. Circulation. 1992;86(1):81-90.

3. Pérez-Castellano N, Garcia EJ, Abeytua M, Soriano J, Serrano JA, Elízaga J, et al. Influence of collateral circulation on inhospital death from anterior acute myocardial infarction. J Am Coll Cardiol. 1998;31(3):512-8.

4. Rentrop KP, Cohen M, Blanke H, Phillips RA. Changes in collateral channel filling immediately after controlled coronary artery occlusion by an angioplasty balloon in human subjects. J Am Coll Cardiol. 1985;5(3):587-92.

5. Kurotobi T, Sato H, Kinjo K, Nakatani D, Mizuno H, Shimizu M, et al. Reduced collateral circulation to the infarctrelated artery in elderly patients with acute myocardial infarction. J Am Coll Cardiol. 2004;44(1):28-34. 
6. Habib GB, Heibig J, Forman SA, Brown BG, Roberts R, Terrin $\mathrm{ML}$, et al. Influence of coronary collateral vessels on myocardial infarct size in humans. Results of phase I thrombolysis in myocardial infarction (TIMI) trial. The TIMI Investigators. Circulation. 1991;83(3):739-46.

7. Sabia PJ, Powers ER, Ragosta M, Sarembock IJ, Burwell LR, Kaul S. An association between collateral blood flow and myocardial viability in patients with recent myocardial infarction. N Engl J Med. 1992;327(26):1825-31.

8. Antoniucci D, Valenti R, Moschi G, Migliorini A, Trapani M, Santoro GM, et al. Relation between preintervention angiographic evidence of coronary collateral circulation and clinical and angiographic outcomes after primary angioplasty or stenting for acute myocardial infarction. Am J Cardiol. 2002;89(2):121-5.

9. Elsman P, van't Hof AW, de Boer MJ, Hoorntje JC, Suryapranata $\mathrm{H}$, Dambrink $\mathrm{JH}$, et al. Role of collateral circulation in the acute phase of ST-segment-elevation myocardial infarction treated with primary coronary intervention. Eur Heart J. 2004;25(10):854-8.

10. Nicolau JC, Nogueira PR, Pinto MA, Serrano CV Jr, Garzon SA. Early infarct artery collateral flow does not improve longterm survival following thrombolytic therapy for acute myocardial infarction. Am J Cardiol. 1999;83(1):21-6.

11. Sorajja P, Gersh BJ, Mehran R, Lansky AJ, Krucoff MW, Webb $J$, et al. Impact of collateral flow on myocardial reperfusion and infarct size in patients undergoing primary angioplasty for acute myocardial infarction. Am Heart J. 2007;154(2):379-84.

12. Goldberg RJ, Gore JM, Alpert JS, Osganian V, de Groot J, Bade J, et al. Cardiogenic shock after acute myocardial infarction: incidence and mortality from a community-wide perspective, 1975 to 1988. N Engl J Med. 1991;325(16):1117-22.

13. Killip $\mathrm{T} 3^{\text {rd }}$, Kimball JT. Treatment of myocardial infarction in a coronary care unit: a two year experience with 250 patients. Am J Cardiol. 1967;20(24):457-64.

14. Hochman JS, Sleeper LA, Webb JG, Sanborn TA, White HD, Talley JD, et al. Early revascularization in acute myocardial infarction complicated by cardiogenic shock. SHOCK Investigators. Should We Emergently Revascularize Occluded Coronaries for Cardiogenic Shock. N Engl J Med. 1999; 341(9):625-34.

15. Hochman JS, Sleeper LA, White HD, Dzavik V, Wong SC, Menon V, et al.; SHOCK Investigators. One-year survival following early revascularization for cardiogenic shock. JAMA. $2001 ; 285(2): 190-2$

16. Kushner FG, Hand M, Smith SC Jr, King SB $3^{\text {rd }}$, Anderson JL, Antman EM, et al. 2009 Focused Updates: ACC/AHA Gui- delines for the Management of Patients With ST-Elevation Myocardial Infarction (updating the 2004 Guideline and 2007 Focused Update) and ACC/AHA/SCAI Guidelines on Percutaneous Coronary Intervention (updating the 2005 Guideline and 2007 Focused Update): a report of the American College of Cardiology Foundation/American Heart Association Task Force on Practice Guidelines. Circulation. 2009; 120(22):2271-306.

17. Babaev A, Frederick PD, Pasta DJ, Every N, Sichrovsky T, Hochman JS; NRMI Investigators. Trends in management and outcomes of patients with acute myocardial infarction complicated by cardiogenic shock. JAMA. 2005;294(4):448-54.

18. Desch S, de Waha S, Eitel I, Koch A, Gutberlet M, Schuler G, et al. Effect of coronary collaterals on long-term prognosis in patients undergoing primary angioplasty for acute ST-elevation myocardial infarction. Am J Cardiol. 2010;106(5):605-11.

19. Smith SC Jr, Feldman TE, Hirshfeld JW Jr, Jacobs AK, Kern MJ, King SB $3^{\text {rd }}$, et al. ACC/AHA/SCAI 2005 Guideline Update for Percutaneous Coronary Intervention-summary article: a report of the American College of Cardiology/American Heart Association Task Force on Practice Guidelines (ACC/AHA/SCAI Writing Committee to Update the 2001 Guidelines for Percutaneous Coronary Intervention). Circulation. 2006;113(1):156-75.

20. TIMI Study Group. The Thrombolysis in Myocardial Infarction (TIMI) trial. Phase I findings. N Engl J Med. 1985;312(14): 932-6.

21. Mattos LA, Lemos Neto PA, Rassi AJ, Marin-Neto JA, Sousa AGMR, Devito FS, et al. Diretrizes da Sociedade Brasileira de Cardiologia - Intervenção Coronária Percutânea e Métodos Adjuntos Diagnósticos em Cardiologia Intervencionista (II Edição - 2008). Arq Bras Cardiol. 2008;91(6 Supl 1):1-58.

22. Hansen JF. Coronary collateral circulation: clinical significance and influence on survival in patients with coronary artery occlusion. Am Heart J. 1989;117(2):290-5.

23. Vyssoulis G, Kyriakidis M, Karpanou E, Kyriakidis C, Sfikakis $\mathrm{P}$, Barbetseas J, et al. A prospective angiographic study of the coronary collateral circulation in coronary arterial disease. Int J Cardiol. 1990;27(2):187-91.

24. Steg PG, Kerner A, Mancini GB, Reynolds HR, Carvalho AC, Fridrich $\mathrm{V}$, et al. Impact of collateral flow to the occluded infarct-related artery on clinical outcomes in patients with recent myocardial infarction: a report from the randomized occluded artery trial. Circulation. 2010;121(25):2724-30.

25. Chatterjee K. Collateral flow to the territory of the occluded infarct-related artery: percutaneous coronary intervention or no percutaneous coronary intervention: why does the gold not always glitter? Circulation. 2010;121(25):2708-10. 